\title{
Úlcera mediofacial secundaria al consumo de cocaína
}

\section{Cocaine induced facial midline destructive ulcer}

Martina Buffetti ${ }^{1}$, Jesica Eliana Radonich ${ }^{2}$, Melanie Reichardt ${ }^{1}$, Eliana Céfalo ${ }^{3}$ y Miguel Ángel Mazzini ${ }^{4}$

\begin{abstract}
RESUMEN
Hay una creciente incidencia de lesiones cutáneas secundarias al consumo de cocaína, con un espectro clínico variado. Esos cuadros tienen un origen multifactorial: el efecto vasoconstrictor de la cocaína, el levamisol que potencia el efecto serotoninérgico y dopaminérgico de esta y el efecto traumático de los cristales al ser inhalado. Aún no se ha logrado consenso para el manejo de esta patología.
\end{abstract}

Se presenta el caso de un paciente varón de 50 años con una úlcera en la mucosa nasal y los tejidos adyacentes, secundaria al consumo de cocaína, que tuvo buena respuesta al tratamiento instaurado.

Palabras clave: cocaína, levamisol, úlcera mediofacial.

Dermatol. Argent. 2020, 26 (3): 121-123

\section{ABSTRACT}

Nowadays there is a growing incidence of skin manifestations due to cocaine abuse with a wide clinical range. Its origin can be traced to multiple factors: the vasoconstrictive effect of the drug, the irritant effects if adulterants like levamisole which induce dopaminergic and serotoninergic responses, and the traumatic effect of the mucosa caused by cocaine crystals insuflated at high velocity. There is yet no consensus on how to handle this pathology.
We report a 50-year-old male patient with an ulcer in nasal mucosa and adjacent tissues, secondary to cocaine use, with good response to treatment.

Key words: cocaine, levamisole, midline facial ulcer.

Dermatol. Argent. 2020, 26 (3): 121-123
1 Médica Residente de Dermatología

2 Médica Jefa de Residentes de Dermatología

${ }^{3}$ Médica de Planta, Servicio de Anatomía Patológica

${ }^{4}$ Jefe del Servicio de Dermatología

Hospital Churruca Visca, Ciudad Autónoma de Buenos Aires, Argentina
Contacto del autor: Martina Buffetti E-mail: martibuffetti@hotmail.com Fecha de trabajo recibido: 9/4/2020 Fecha de trabajo aceptado: 4/9/2020

Conflicto de interés: los autores declaran que no existe conflicto de interés.

\section{CASO CLÍNICO}

Un varón de 50 años, con antecedentes de hipertensión arterial, dislipidemia, sedentarismo y tabaquista activo, consultó en el Servicio de Otorrinolaringología de nuestro hospital por una lesión de un año de evolución en la mucosa nasal, que se extendía al filtrum $\mathrm{y}$ al labio superior.

En el examen físico se evidenciaba una úlcera dolorosa, de bordes eritematosos infiltrados sobreelevados, con fondo granuloso y sectores cubiertos de fibrina y de costras hemáticas (Foto 1). Además, presentaba una perforación septal y rinorrea mucopurulenta abundan- te. No refería fiebre, equivalentes febriles ni otra sintomatología sistémica.

Los diagnósticos presuntivos planteados fueron: infección de piel y partes blandas, lesión destructiva mediofacial inducida por cocaína, granulomatosis con poliangitis (Wegener), linfoma nasal de células T/NK, histoplasmosis y leishmaniasis mucocutánea.

Se solicitaron múltiples exámenes complementarios e imagenológicos para evaluar la extensión de la lesión. Tanto la rinofibroscopia rígida como la tomografía computada (TC) del macizo craneofacial confirmaron la pre- 
sencia de una perforación septal. La resonancia magnética (RM) con contraste intravenoso puso en evidencia una imagen heterogénea en las fosas nasales, hipointensa en T2, que no se realzaba tras la administración del contraste. En los estudios de laboratorio se observó elevación de los reactantes de fase aguda (VSG, PCR), positividad para p-ANCA y negatividad para FAN, anti-Ro, anti-La, mieloperoxidasa (MPO) y proteinasa 3 (PR3). La TC de tórax y la orina de 24 horas no presentaron alteraciones.

El examen directo y los cultivos para bacterias, hongos y leishmaniasis fueron negativos, por lo que se descartaron las patologías infecciosas.

La histopatología evidenció una mucosa nasal con metaplasia escamosa parcial, lámina propia con fibrosis, plasmocitos dispersos y arteriolas con fibrosis concéntrica de su pared y trombosis luminal canalizada (Foto 2). Se interpretó como rinitis crónica inespecífica con arteriopatía oclusiva sin actividad.

Después de haber descartado la mayoría de los diagnósticos presuntivos y al tener en cuenta que la presencia de p-ANCA se asocia al consumo de cocaína adulterada con levamisol, se decidió reinterrogar al paciente acerca de sus hábitos toxicológicos y se realizó la determinación de cocaína en la orina, que fue positiva. Se arribó al diagnóstico de lesión destructiva (úlcera) de la línea mediofacial por cocaína.

El tratamiento instaurado fue la limpieza quirúrgica con oclusión de la lesión, asociada a meprednisona 40 $\mathrm{mg} /$ día y el seguimiento conjunto con el Servicio de psiquiatría. Se logró el abandono del consumo de cocaína, hábito que el paciente tenía desde hacía 30 años. Tras un mes de tratamiento y con el descenso gradual de la meprednisona, presentó una franca mejoría con reepitelización de la lesión (Foto 3).

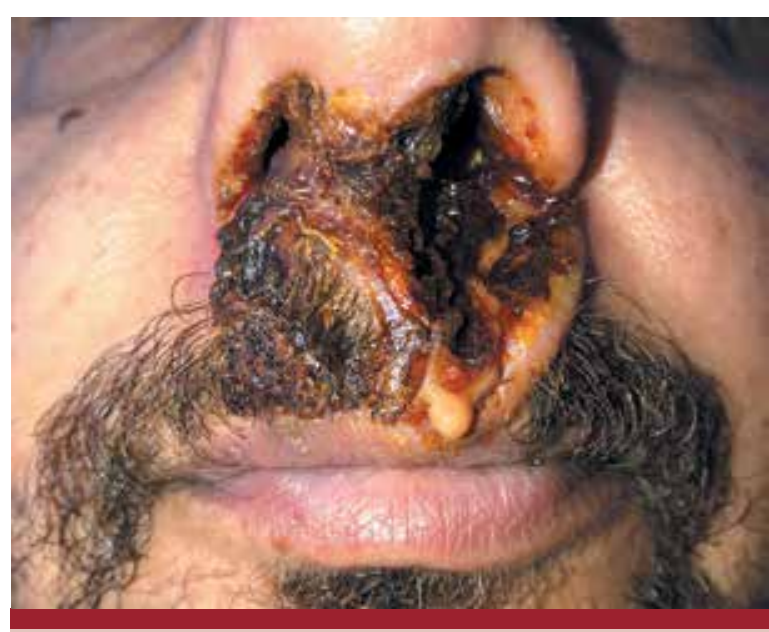

F0T0 1: Úlcera dolorosa de bordes eritematosos, inflitrados sobreelevados y lecho granuloso, con sectores cubiertos de fibrina y costras hemáticas.

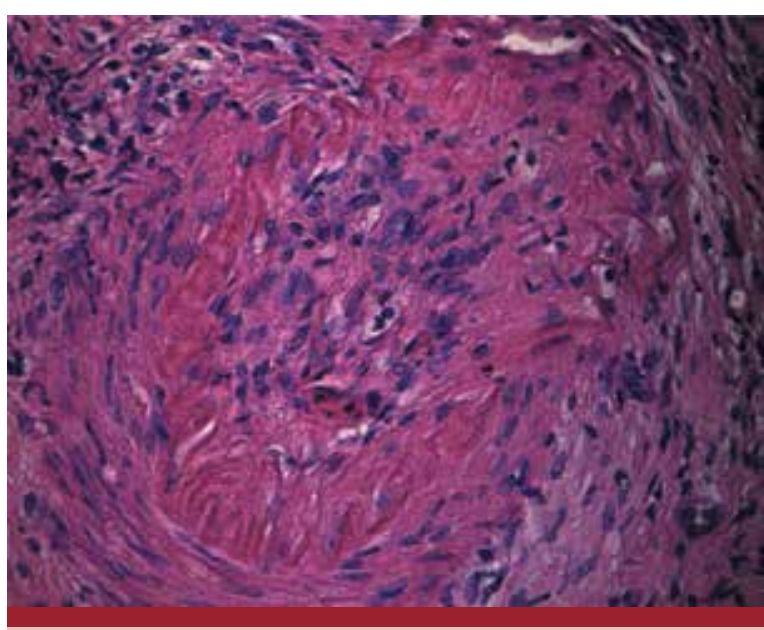

FOTO 2: Arteriola con fibrosis concéntrica de su pared y trombosis luminal canalizada (HyE, 40X).

F0T0 3: Reepitelización de la lesión al mes de tratamiento.

\section{COMENTARIOS}

Existe una creciente incidencia de lesiones secundarias al consumo de cocaína ${ }^{1}$. Tienen un espectro muy amplio, desde úlceras necrosantes, perforación septal, enfermedades ampollares, hasta vasculitis de pequeños y medianos vasos, principalmente en las orejas, la punta nasal, las mejillas y las extremidades, entre otras ${ }^{2,3}$.

La fisiopatología es multifactorial. El efecto vasoconstrictor de la cocaína genera necrosis isquémica local en los sitios de contacto con la droga, por lo que pueden afectarse estructuras de la nariz, los senos nasales y el paladar. También se debe tener en cuenta el efecto traumático en la mucosa, producto de los cristales de cocaína inhalados a alta velocidad ${ }^{3}$. Por otro lado, algunos autores relacionan la trombosis microangiopática y agranulocitosis/neutropenia con el levamisol, adulterante agregado a la cocaína ${ }^{4}$. Se han planteado múltiples teorías del efecto del levamisol sobre esta patología. Una de ellas describe que puede potenciar los efectos colinérgicos de los receptores 
nicotínicos en el sistema nervioso central. Otra señala que el levamisol se metaboliza a aminorex, un sustrato de los transportadores de serotonina, por lo cual actuaría indirectamente como agonista serotoninérgico ${ }^{4}$.

La vasculitis por levamisol secundaria al consumo de cocaína se describió por primera vez en 2010. Clínicamente, se caracteriza por lesiones cutáneas de espectro variado, agranulocitosis/neutropenia y ANCA positivos. Suele verse en los pacientes con más de un año de consumo crónico de cocaína, como en este caso ${ }^{4}$.

Se arriba al diagnóstico al tener en cuenta la clínica, el laboratorio, la histopatología y los antecedentes de consumo de cocaína. Los estudios de laboratorio pueden evidenciar leucopenia o neutropenia en $50-60 \%$ de los casos y reactantes de fase aguda elevados (proteína C-reactiva y eritrosedimentación $)^{4,5}$. Asimismo, el ANCA positivo, que suele tener un patrón de inmunofluorescencia perinuclear ( $\mathrm{p}$-ANCA) y especificidad antigénica hacia la elastasa neutrofílica humana (HNE). Esto ayuda a diferenciarlo de la granulomatosis con poliangitis (Wegener), que presenta también ANCA positivo, pero con patrón citoplasmático (c-ANCA) y especificidad hacia la proteinasa $3(\mathrm{PR} 3)^{2,5}$. Otro hallazgo clínico para diferenciar estas dos entidades es el paladar perforado, el cual se presenta con mayor frecuencia en los cuadros asociados al abuso de cocaína que a la granulomatosis con poliangitis (Wegener) ${ }^{6,7}$.

En la histopatología se pueden observar cambios inespecíficos como vasculitis leucocitoclástica; infiltra-

\section{BIBLIOGRAFÍA}

1. Salas-Espíndola Y, Peniche-Castellanos A, López-Gehrke I, Mercadillo-Pérez P. Vasculitis leucocitoclástica asociada a consumo de cocaína. Actas Dermosifilogr 2011;102:825-827.

2. Barrera OM, De la Rivera VM, Vela UJ, Barrera MR, et ál. Lesiones destructivas de la línea media facial secundarias al consumo de cocaína. Caso clínico. Rev Med Chile 2018;146:1070-1073.

3. Trimarchi M, Gregorini G, Facchetti $\mathrm{F}$, Morassi ML, et ál. Cocaine-induced midline destructive lesions: clinical, radiographic, histopathologic, and serologic features and their differentiation from Wegener granulomatosis. Medicine (Baltimore) 2001;80:391-404.

4. Calligaris M, Nipoti J, Gorosito M, Garrido MG. Vasculitis inducida por cocaína. Dermatol Argent 2017;23:196-198. do inflamatorio de células mononucleares, neutrófilos o eosinófilos; trombosis microangiopática, y necrosis fibrinoide ${ }^{2,3}$. El infiltrado inflamatorio suele encontrarse en las paredes de vénulas o arteriolas, lo que da lugar a una disminución del calibre de estas ${ }^{3}$.

Entre los diagnósticos diferenciales de las lesiones destructivas de la línea media se encuentran granulomatosis con poliangitis, linfoma T/NK, sarcoidosis, leishmaniasis e histoplasmosis, todos descartados en este paciente ${ }^{4}$.

El éxito terapéutico depende de la suspensión del consumo de cocaína. Se ha observado que en casos iniciales la abstinencia puede normalizar la mucosa y revertir el proceso patológico. También se describieron tratamientos locales conservadores como el desbridamiento del tejido necrótico, los lavados con solución salina y los antibióticos locales o sistémicos ${ }^{8}$. Debe existir un período mínimo de abstinencia de 6 a 12 meses para plantear procedimientos quirúrgicos reconstructivos $^{2}$.

El interés del caso analizado es comunicar una patología de creciente incidencia, que suele ser difícil de diferenciar de otras enfermedades sistémicas que comprometen la mucosa nasal y los senos paranasales. A la vez, resaltar la buena respuesta al uso de los corticosteroides del paciente presentado, ya que no hay consenso para el manejo de estos casos, además de la importancia del interrogatorio cuando nos enfrentamos a patologías por abuso de sustancias.

5. Roverano S, Gallo J, Ortiz A, Paira S. Manifestaciones sistémicas asociadas al consumo de cocaína: comunicación de un caso. Rev Argent Reumatol 2016;27:50-52.

6. Trimarchi M, Bondi S, Della Torre $\mathrm{E}$, Terreni MR, et ál. Palate perforation differentiates cocaine-induced midline destructive lesions from granulomatosis with polyangiitis. Acta Otorhinolaryngol Ital 2017;37:281-285.

7. Fernández Blanco G, Madeo MC, Martínez M, Vázquez ME. Case for diagnosis. Palate perforation due to cocaine use. An Bras Dermatol 2017;92:877-878.

8. Trimarchi M, Bussi M, Sinico RA, Meroni P, et ál. Cocaineinduced midline destructive lesions - An autoimmune disease? Autoimmun Rev 2013;12:496-500. 